\title{
Neuro-Interventions for the Neonates with Brain Arteriovenous Fistulas: With Special Reference to Access Routes
}

\author{
Masaki KOMIYAMA, ${ }^{1}$ Aiko TERADA, ${ }^{1}$ and Tomoya ISHIGURO ${ }^{1}$ \\ ${ }^{1}$ Department of Neuro-Intervention, Osaka City General Hospital, Osaka, Osaka
}

\begin{abstract}
Neonatal neuro-intervention is challenging. The purpose of this article is to report the neuro-intervention for the neonates with brain arteriovenous fistulas (AVFs), with special reference to access routes. Fifteen neonates ( 12 boys and 3 girls) who underwent neuro-intervention within the first 14 days of life were included. Their diagnoses included vein of Galen aneurysmal malformation (6), dural sinus malformations with arteriovenous (AV) shunts (6), pial AVF (2), and epidural AVF (1). Birth weight ranged from 1,538 g to $3,778 \mathrm{~g}$ (mean $2,525 \mathrm{~g}$ ). Neuro-interventions, especially access routes, in the neonatal periods $(<1$ month) were retrospectively reviewed. All neonates presented with severe cardiac failure. In total, 29 interventions (mean 1.9) were performed within 1 month. Although 12 neonates with birth weight more than 2,700 $\mathrm{g}$ could be treated through transfemoral arterial routes, 3 neonates with birth weight less than $2,200 \mathrm{~g}$ could not be treated successfully by femoral arterial routes. Interventions were performed through 19 femoral arterial, 3 femoral venous, 2 umbilical arterial, 3 umbilical venous, 3 transcardiac, and 2 direct carotid routes. Their overall outcomes were six good recovery, one moderate disability, two severe disabilities, one vegetative state, and five deaths with a mean follow-up period of 7 years 2 months. Neuro-intervention for the neonates with birth weight more than $2,700 \mathrm{~g}$ can be performed by femoral arterial routes using a $4 \mathrm{~F}$ sheath. For those with birth weight less than $2,200 \mathrm{~g}$, however, alternative access routes are required.
\end{abstract}

Key words: access route, arteriovenous fistula, body weight, neonate, neuro-intervention

\section{Introduction}

Brain arteriovenous fistulas (AVFs) encountered in the neonatal period include vein of Galen aneurysmal malformation, dural sinus malformation with AV shunts, and less frequently pial AVF. ${ }^{1-5)}$ Whatever brain AVFs the neonates had in this period, they commonly present with severe congestive heart failure. ${ }^{6)}$ Treatment is directed to ameliorate cardiac failure by reduction of shunt flow in addition to aggressive medical treatment. Direct surgery has no longer any role in treatment of such neonates with severe heart failure. ${ }^{7)}$ Neuro-intervention has an important role to achieve this goal. ${ }^{8,9}$ Brain damage at birth caused by brain AVFs is contraindication of treatment in Europe and in the United States, ${ }^{3,5}$ but therapeutic abstention for such neonates is not always possible in Japan when the parents strongly wish to treat the affected baby. Further, prediction of the outcome in the early neonatal period is not always reliable. ${ }^{10)}$ Transcatheter treatment is not

Received December 17, 2015; Accepted January 11, 2016 straightforward in this period due to fragilities of the cerebrovascular system of neonates, which include difficulty of catheter access. The purpose of this study is to report the neuro-intervention for the neonates with brain AVFs, with special reference to the vascular access routes.

\section{Materials and Methods}

Fifteen neonates (12 boys and 3 girls) who underwent the initial neuro-interventions within the first 14 days of life were included in this study. Indication of treatment for these 15 neonates was severe congestive heart failure and respiratory failure. All neonates were intubated and ventilated when we considered neuro-interventions of brain AVFs. Neonatologists judged from clinical status and results of ultrasound examination that medical treatment could not control their heart failure due to a large amount of AV shunts. Focal neurological deficits including hemiparesis were not apparent in all cases. No patients had congenital cardiac anomalies. Five neonates with cerebral AV shunts without severe 
heart failure during the study period were excluded from this study and their diagnoses included three veins of Galen aneurysmal malformations and two dural sinus malformations with AV shunts. They were treated in 3-5 months after birth. Diagnoses of 15 patients included six veins of Galen aneurysmal malformation (all choroidal types), six dural sinus malformations with AV shunts (all medial types involving the torcular Herophili), two pial AVFs, and one epidural AVF at the craniocervical junction including the hypoglossal canal and intramuscular AVF in the cervical muscles presenting as Cobb syndrome. The main feeding arteries in two neonates with pial AVFs were middle cerebral artery and basilar artery, respectively. The purpose of the interventions in this period was not the anatomical cure, but to improve the clinical status. They were treated in the period between November 1995 and March 2015 and were all followed-up until September 2015 except for the dead (Table 1). All procedures were performed after obtaining written informed consents from the patient's parents.

Timing of delivery was at the gestational periods between 31 weeks 0 day and 41 weeks 6 days (mean 37 weeks 1 day). Modes of delivery were two spontaneous deliveries and 13 caesarian sections. Antenatal diagnoses of brain AVFs by fetal magnetic resonance (MR) imaging were established in 10 neonates. Birth weight ranged from 1,538 g to 3,778 g (mean 2,525 g). There was no neonate with birth weight between $2,200 \mathrm{~g}$ and $2,700 \mathrm{~g}$. Ten neonates were treated in our hospital and the remaining five were treated in the outside hospitals by the senior author (M.K.). Brain damages (atrophy, cerebromalacia, hemorrhage, and infarction) on pre-treatment ultrasound and computed tomography (CT)/MR images were observed in six neonates (Cases 2, 4, 10, 11, 13, 14), and no apparent brain damages were observed in the remaining nine neonates.

Femoral arterial approach under general anesthesia was principally used if feasible. When femoral arterial approach was not possible, the other approaches including umbilical approaches were deliberately selected. For transfemoral arterial and venous approaches and transumbilical arterial approach, a $4 \mathrm{~F}$ sheath (7-10 cm long) was used, and a $4 \mathrm{~F}$ diagnostic catheter (60-100 cm long) was used as a guiding catheter. For transumbilical venous approach, a 4-5F sheath (7-10 cm long) was used and a $4 \mathrm{~F}$ diagnostic catheter $(60-100 \mathrm{~cm}$ long) was used as a guiding catheter. For transumbilical or transfemoral venous transcardiac approach, a 5-6F sheath (7-10 cm long) was used, and a Tracker 38 catheter $(105 \mathrm{~cm}$ long, Boston Scientific, Fremont, USA), 4-5F Cerulean catheter (125 cm long, Medikit,
Tokyo), or 4F Fubuki catheter (120 cm long, Asahi Intecc Co., Ltd., Nagoya) was used as transcardiac guiding catheters (Fig. 1). For direct carotid arterial approach, an 18-G elastic needle was inserted into the common carotid artery under vision. As a coaxial microcatheter to deliver embolic materials, a Magic catheter (Balt, Montmorency, France) was used for glue embolization and variable 18-type microcatheters were used for detachable coil and/or glue embolization. Pediatric anesthesiologists performed general anesthesia in all cases with endotracheal intubation. Arterial blood pressure and oxygen saturation were monitored throughout the procedures. Embolic materials in the arterial embolization were mostly glue, i.e., mixture of N-butyl cyanoacrylate (NBCA) and Lipiodol (Guerbet, France) in addition to variable sizes and shapes of 10- or 14-type electrically detachable platinum coils. Those in the venous embolization were 14- or 18-type electrically detachable platinum coils. Coils were used for scaffolding a glue cast in the feeding arteries by transarterial approach or for occlusion of the dural ectasia by transvenous approach. NBCA concentrations were above $33 \%$, but high concentration such as $60-80 \%$ was required to occlude high flow fistulas in the neonatal interventions. Outcomes were classified as good recovery (GR) when the patient developed almost normally, moderately disabled (MD) when the patient grew with moderate developmental delay, severely disabled (SD) when the patient is severely handicapped, and death (D). Clinical symptoms, neuro-interventions especially vascular access routes, and outcomes were reviewed retrospectively.

\section{Results}

All 15 neonates presented with severe cardiac failure due to volume overload attributable to the brain AVFs. No neonate had cardiac anomalies. Apgar scores at 1 min and 5 min were 2-9 and 6-9, 6.0 and 7.9 (mean), respectively. Thus, Apgar scores at birth were not always correlated to the severity of heart failure. Although the general conditions were poor due to heart failure, focal neurological deficits were not observed in any neonates as were the case with neonates with pediatric stroke in this period. Initial interventions were required on day 0 (3 neonates), on day 1 (3 neonates), on day 2-6 (5 neonates), and on day 7-9 (4 neonates) with a mean of day 3.7. The earliest intervention was performed 4 hours after caesarian section in Case 5 . Three neonates with birth weight less than $2,200 \mathrm{~g}$ could not be treated successfully by femoral arterial routes. In one boy with body weight of $1,728 \mathrm{~g}$ at birth and 1,463 $\mathrm{g}$ at intervention on day 7 (Case 2), vascular sheath insertion caused dissection/laceration 


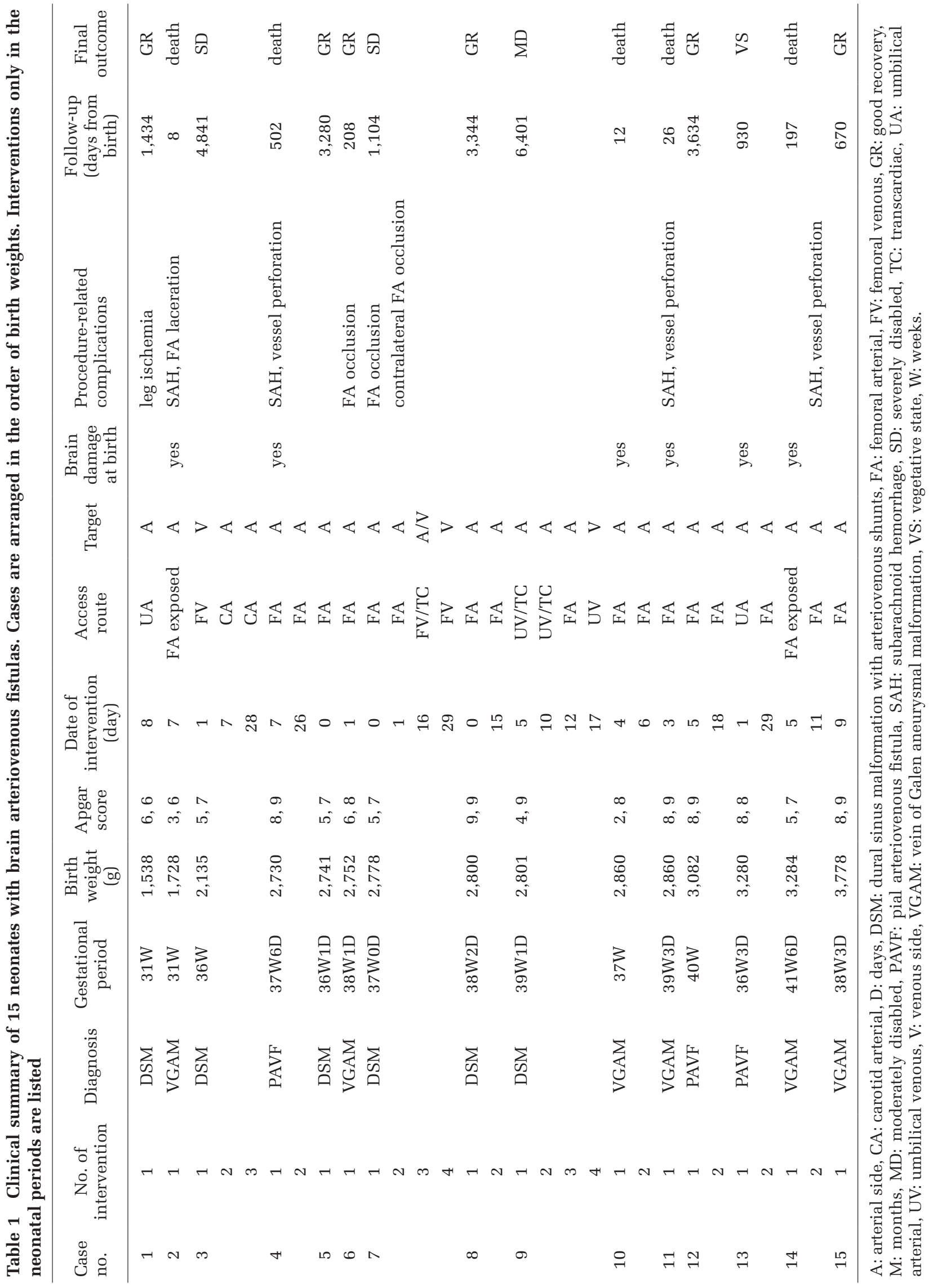




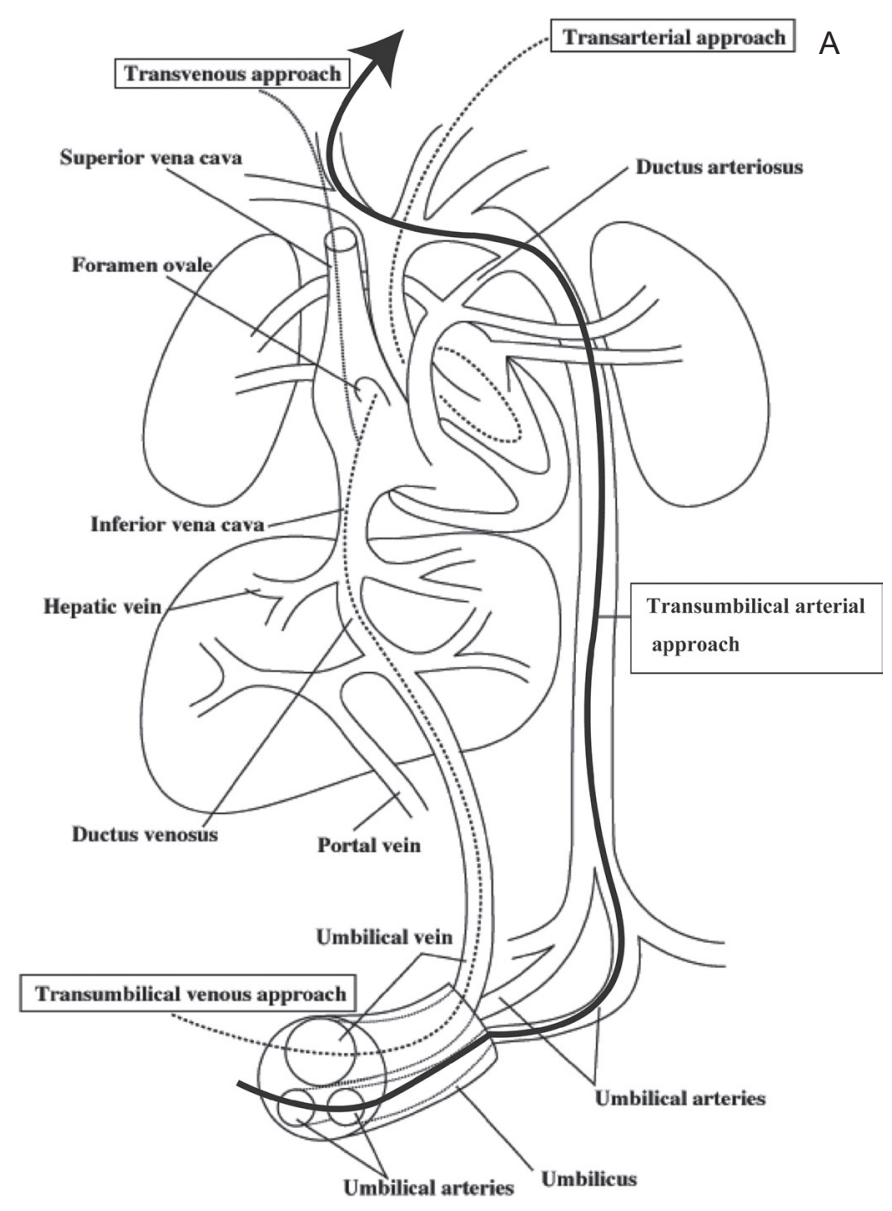

of the femoral arteries bilaterally, and finally both arteries were ligated. The remaining two neonates (Cases 1 and 3) were treated through various routes, such as femoral venous and umbilical arterial routes (Figs. 2, 3). Twelve neonates with birth weight more than 2,700 g could be treated through femoral arterial routes, but for one neonate in whom bilateral femoral arteries were occluded by the first two interventions (Case 7), femoral venous and transcardiac routes had to be used in the subsequent interventions (Fig. 4.)

Twenty-nine interventions in total consisted of 25 arterial, 3 venous, and 1 both arterial/venous embolization through 19 femoral arterial, 3 femoral venous, 2 umbilical arterial, 3 umbilical venous, 3 transcardiac (femoral or umbilical venous), and 2 direct carotid arterial routes. Excluding the lethal cases, AV shunts were disappeared or not visualized on the following MR angiography in all cases except for Cases 7 and 15, in which further treatment were scheduled for the small residual AV shunts.

Complications included leg ischemia (1), laceration or occlusion of femoral arteries (3), and intraoperative hemorrhage including intracranial vessel rupture and perforation (4). Leg ischemia after umbilical arterial

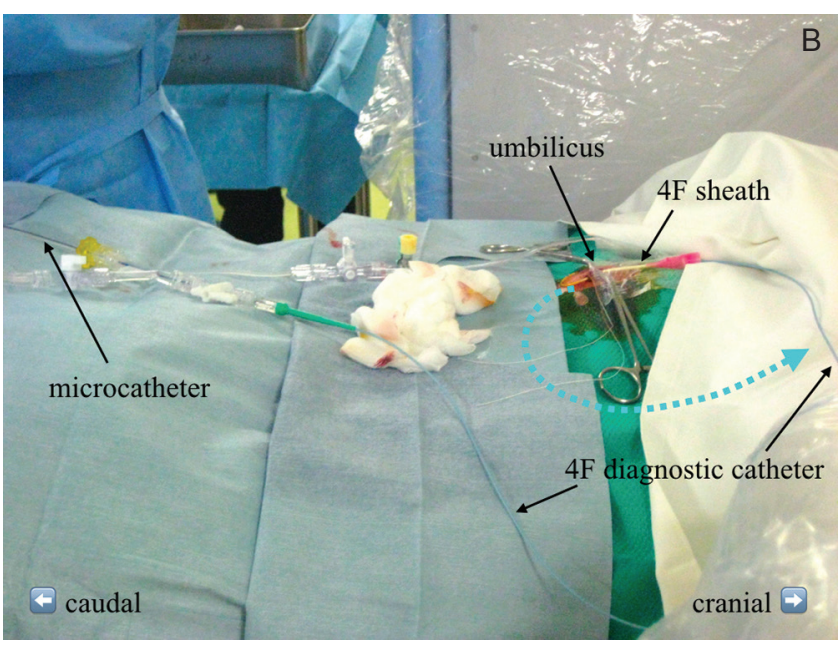

Fig. 1 Transumbilical approaches. A: Transumbilical arterial approach is shown as a solid line with an arrowhead. Transumbilical venous, transcardiac arterial approach is shown as an interrupted line while transumbilical venous, jugular venous approach is depicted as a fine interrupted line. (Modified illustration from Journal of Neurosurgery with permission. ${ }^{14)}$ ) B: Intraprocedural photograph of the transumbilical arterial approach (Case 1). The microcatheter and diagnostic catheter are coaxially introduced into the $4 \mathrm{~F}$ sheath in the umbilicus. Interrupted blue line indicates an expected course of the catheter system through the umbilical artery - internal iliac artery - common iliac artery - descending aorta. The patient's head is on the right side of the figure.

approach responded well to medical treatment and recovered completely (Case 1). Unilateral and bilateral femoral artery occlusion in two cases (Cases 6 and 7) did not cause leg ischemia, but forced to use alternative access routes for the subsequent interventions in the latter case. Intra-procedural hemorrhages resulted in four deaths. Post-procedural hemorrhage after the third intervention at 7 months resulted in one vegetative state (Case 13).

Their overall outcomes were six good recovery, one moderate disability, two severe disability, one vegetative state, and five deaths. Outcomes of six neonates with pre-treatment brain damages on CT/MR were five deaths and one vegetative state. Follow-up period counted from birth ranged from 7 months to 17 years 9 months (mean 7 years 2 months) except for the lethal cases.

\section{Discussion}

\section{Neonates with high-flow brain AVFs}

High-flow brain AVFs in the neonatal periods include vein of Galen aneurysmal malformation, dural sinus malformation with AV shunts, and less 

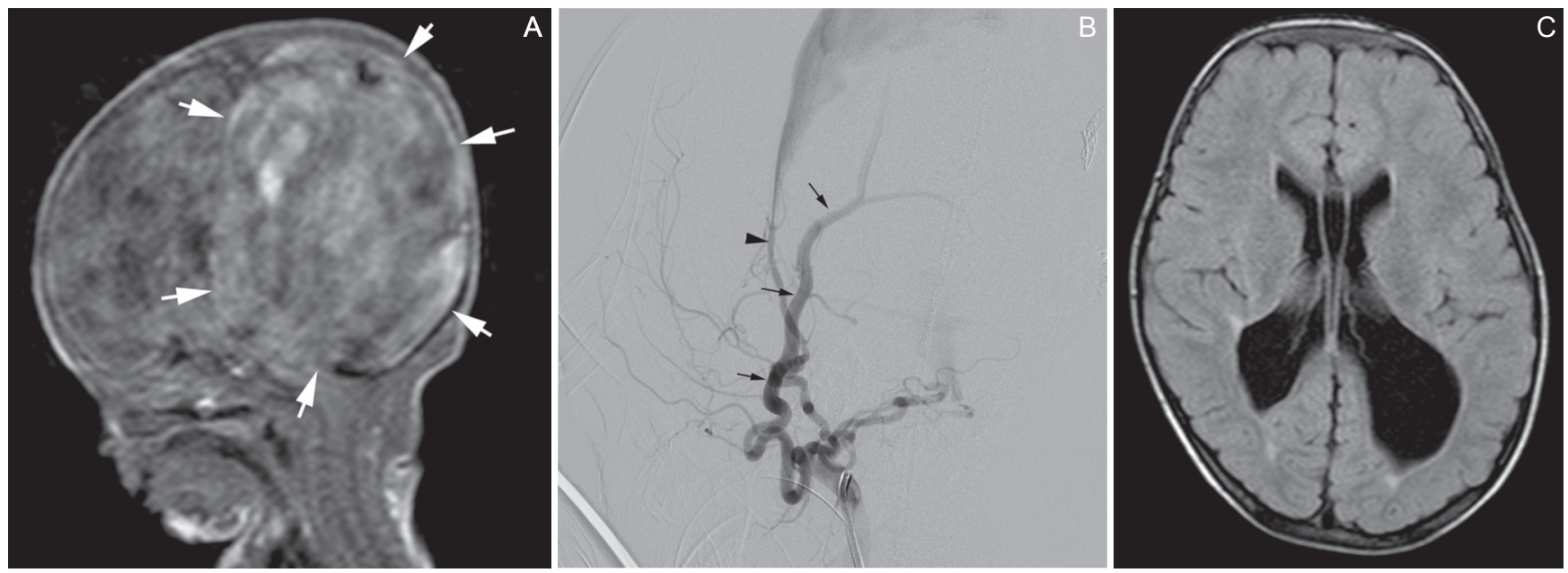

Fig. 2 Case 1, a girl with birth weight of 1,538 g. Transumbilical arterial approach for dural sinus malformation with arteriovenous (AV) shunts. A: $T_{1}$-weighted sagittal magnetic resonance (MR) image on day 1 shows markedly dilated dural ectasia at the torcular herophili (arrows). B: Left common carotid injection from a $4 F$ catheter introduced from right umbilical artery. Arrows indicate large left middle meningeal artery, which was embolized with glue. Arrowhead points at left tentorial artery. C: Fluid-attenuated inversion recovery image obtained at the age of 2 years and 6 months shows no brain damage or dural ectasia at the torcular herophili. MR angiography (not shown) demonstrates no AV shunt.
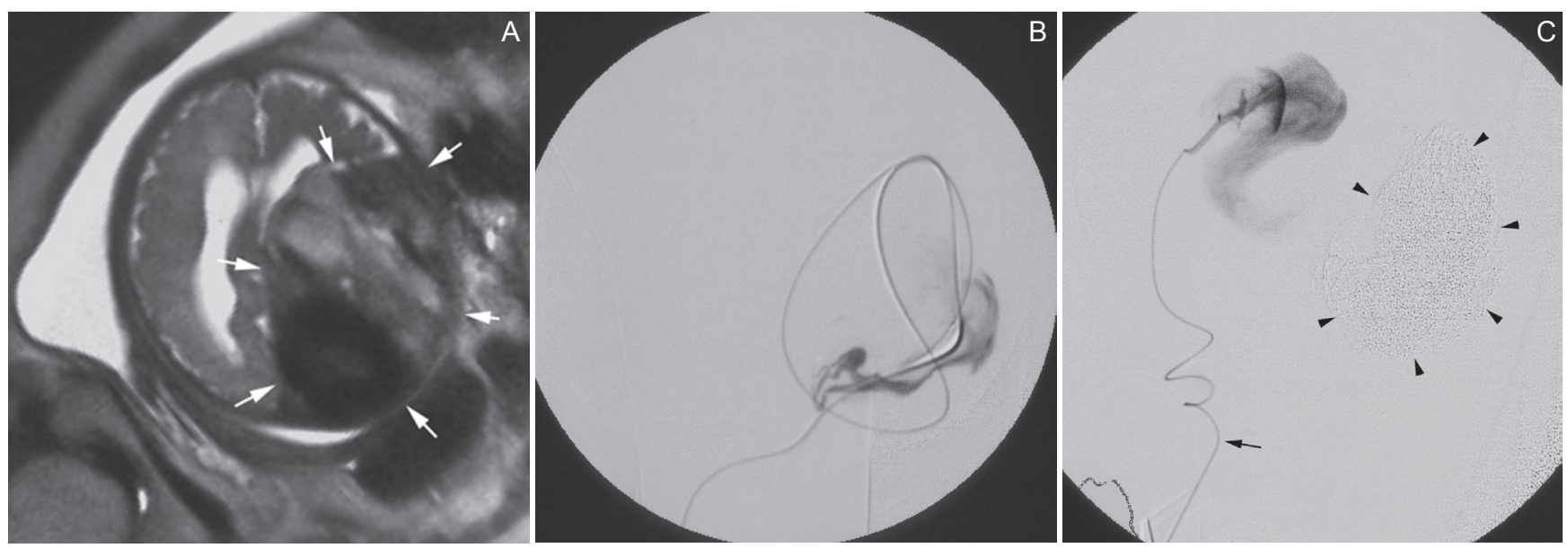

Fig. 3 Case 3, a boy with birth weight of 2,135 g. Femoral venous approach and direct carotid arterial approach for dural sinus malformation with arteriovenous shunts. A: Fetal $\mathrm{T}_{2}$-weighted axial magnetic resonance image obtained 2 days before delivery shows dilated dural ectasia at the torcular herophili (arrows). B: Lateral venography of markedly dilated dural ectasia on day 1. Transfemoral venous embolization to the dural ectasia was performed without angiographic control. Large amount of coils was deposited in the ectasia. C: On day 7, left common carotid artery was surgically exposed, and direct puncture of an 18-gauge elastic needle allowed direct carotid arterial access. Needle tip (arrow) was in the left external carotid artery. Selective injection of left middle meningeal artery (lateral view) was followed by glue embolization. Arrowheads indicate the previously deposited coil mass in dilated dural ectasia.

frequently pial AVF.1-5) Their symptoms in this period are inevitably severe congestive heart failure. ${ }^{6,8)}$ If heart failure is so severe during the fetal period, fetal death in utero occurs due to hydrops fetalis. Soon after delivery, ultrasound evaluation of the cardiac function may prompt early intervention often within the first few days of life, occasionally, on the day of delivery in some case. Actually in our series, neonates were treated initially on day 3.7 in average. Birth weights may indicate maturity of neonates, which define the availability of the vascular access for intervention. Large AV shunts 


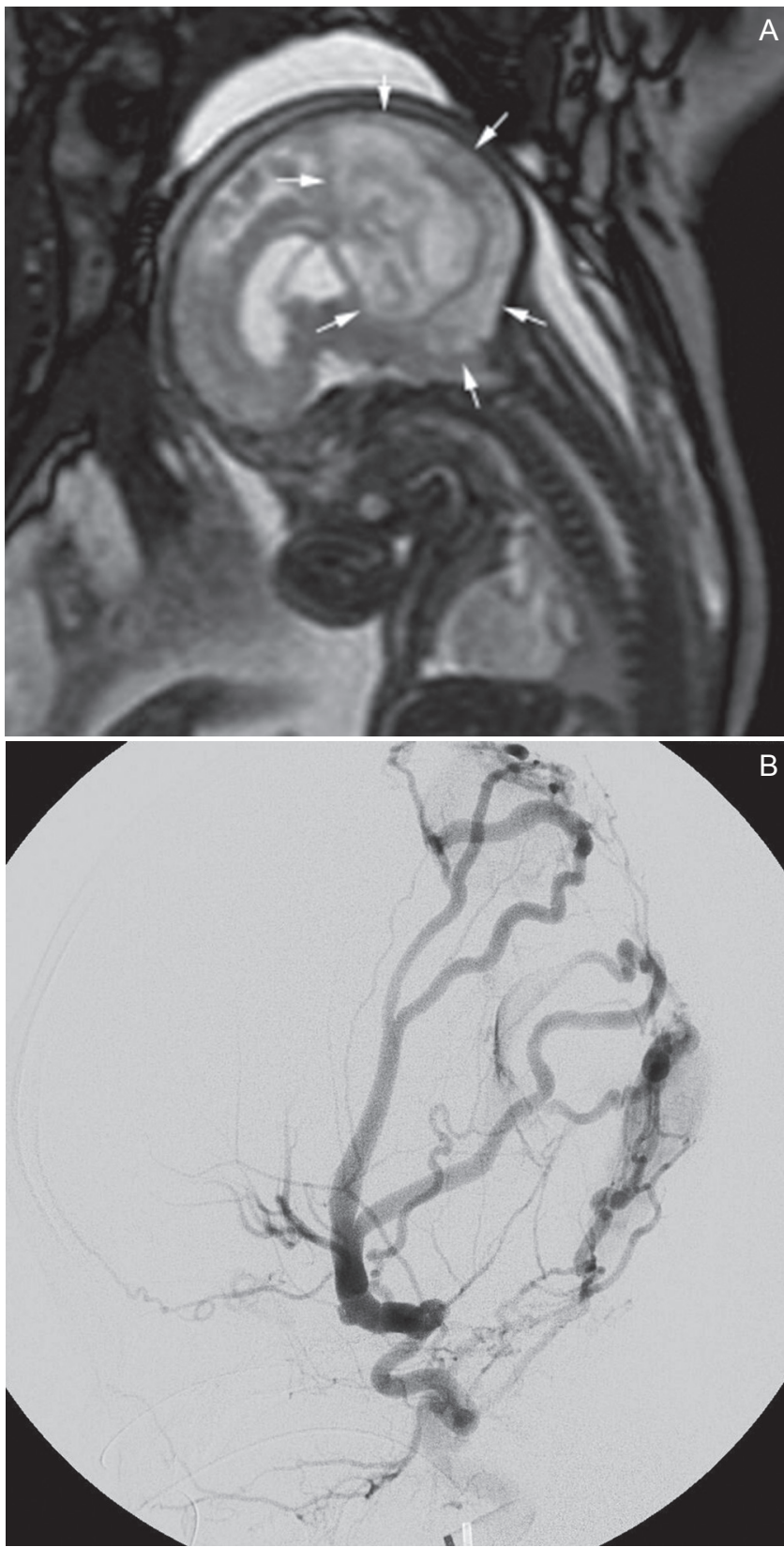

in the brain are associated with compromised blood flow in the descending aorta. Consequently, blood flow to the femoral arteries is also compromised. Even under this condition, femoral arterial approach could be applied for neonates with birth weight more than 2,700 g. However, neonates with birth weight less than $2,700 \mathrm{~g}$ carry a risk of occlusion of femoral artery even if treatment could be successfully performed. In fact, insertion of a $4 \mathrm{~F}$ sheath to the femoral arteries of the neonate with birth weight of 1,728 $\mathrm{g}$ under direct vision resulted in dissection/laceration of bilateral femoral arteries (Case 2). Similarly, a $4 \mathrm{~F}$ sheath insertion resulted in bilateral femoral arteries occlusion in a neonate with birth

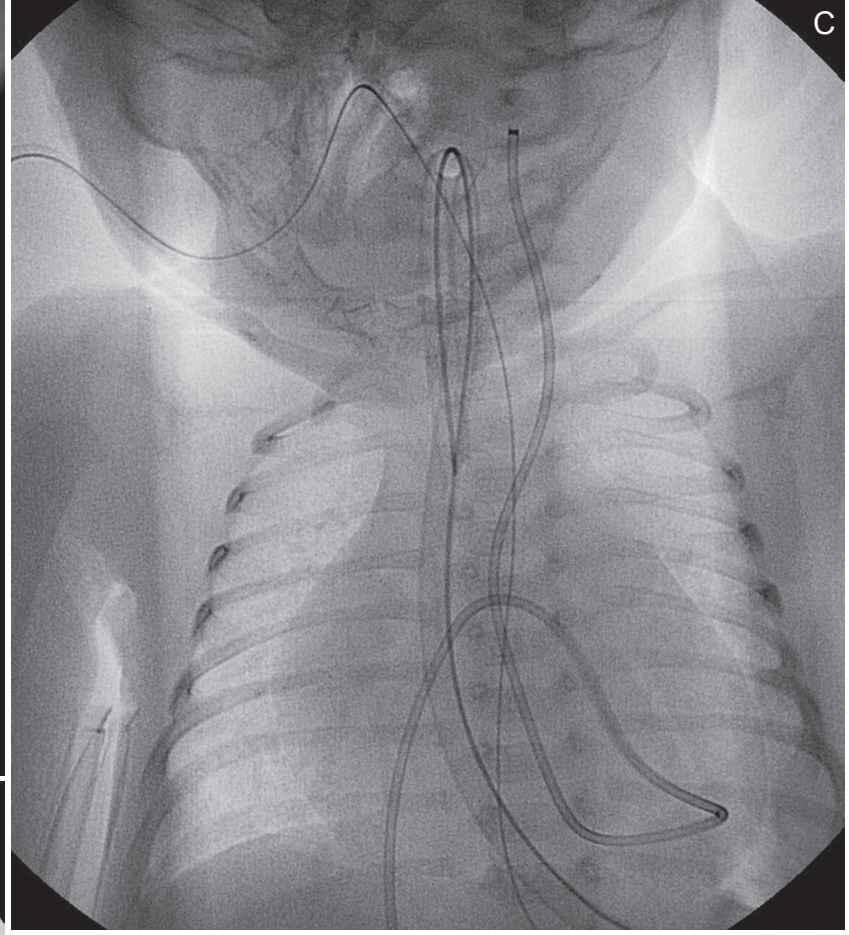

Fig. 4 Case 7, a boy with birth weight of 2,778 g. Transfemoral venous, transcardiac approach for dural sinus malformation with arteriovenous shunts. A: Fetal $T_{2}$-weighted sagittal magnetic resonance image obtained 10 days before delivery shows dilated dural ectasia at the torcular herophili (arrows). B: On day o, right lateral external carotid angiography shows dilated middle meningeal arteries. Femoral arterial embolization to the middle meningeal arteries was performed. C: On day 16, due to occlusion of bilateral femoral arteries after the first and second interventions, right femoral venous, transcardiac approach was required. Chest $\mathrm{X}$-ray shows a $4 \mathrm{~F}$ catheter navigated from right femoral vein through right atrium, left atrium, left ventricle, and ascending aorta to common carotid artery. This approach enabled arterial embolization of many dural feeders. Cardiomegaly with a cardiothoracic ratio of $73 \%$ is apparent.

weight of 2,778 g (Case 7). In neonates with birth weight less than $2,200 \mathrm{~g}$, secure femoral arterial access is not anatomically possible. Consequently, the other access routes have to be selected. These might be umbilical access, transcardiac access, or direct carotid arterial access at the neck.

\section{Transfemoral arterial approach}

For neonates with severe heart failure, transfemoral arterial approach is a standard practice. ${ }^{5}{ }^{5}$ Catheterization to femoral artery in neonates is challenging and is associated with thromboembolic complications and subsequent occlusion of the femoral arteries. ${ }^{11}$ However, femoral arterial access is generally possible, 
in our experiences, for neonates with birth weight more than 2,700 g. Femoral arterial sheath could be left in femoral artery for the subsequent intervention within several days, but this method is not recommended. ${ }^{12)}$ Instead, the sheath should be removed soon after the intervention to avoid ipsilateral leg ischemia. All femoral arteries inserted with $4 \mathrm{~F}$ sheaths lose antegrade blood flow during the procedures, but collateral flow supplies the distal segment of femoral arteries immediately. Temporary occlusion of femoral artery with a vascular sheath does not cause serious leg ischemia, but prolonged engagement of the sheath might cause compromised flow to the ipsilateral leg. Although occlusion of femoral artery at the puncture site does not always lead to the leg ischemia or chronic shortening of the leg, loss of the ipsilateral femoral arterial access for the subsequent interventions is present.

\section{Transumbilical arterial approach}

Umbilical arterial access for neuro-intervention was first introduced by Alex Berenstein in 1997. ${ }^{13)}$ This approach allows catheter access even for neonates with birth weight less than 2,000 g. Actually, Berenstein et al. treated a neonate with birth weight of 1,930 g. Umbilical route can be used within several days after birth. It is recommended to catheterize both umbilical artery and vein soon after delivery whenever possible for possible transumbilical approach in the cases with brain AVFs. ${ }^{14-16)}$ Umbilical arterial approach has, however, an inherent risk of vessel perforation, thromboembolic complications including leg ischemia, infection, aortic aneurysm, air embolism, hemorrhage, bladder rupture, and intestine perforation. ${ }^{17,18)}$ Using this approach, a neonate with dural sinus malformation with birth weight of 1,538 $\mathrm{g}$ was successfully treated. ${ }^{16)}$ When umbilical arterial and femoral arterial accesses are not feasible, the last resorts are as follows: (1) direct carotid arterial access at the neck, (2) femoral venous access, (3) umbilical venous access, and (4) internal jugular venous access.

\section{Direct carotid arterial approach}

Carotid arteries are usually large due to increased blood flow due to AV shunts. We performed direct carotid arterial access in a neonate with birth weight of 2,135 g by small surgical exposure of cervical common carotid artery and puncturing it with an 18-G needle under direct vision with the help of pediatric cardiac surgeons (Case 3). Percutaneous puncture could be performed under ultrasound monitoring, but it has a risk of puncturing the arterialized internal jugular vein and any suboptimal puncture of common carotid artery. Drawback of this carotid approach is that this allows only ispilateral carotid access. ${ }^{19)}$ The contralateral carotid arterial access requires the other puncture of the carotid artery in the separate session.

\section{Transvenous approaches (femoral, umbilical, and jugular venous approaches)}

The purely venous approaches include umbilical venous, femoral venous, and jugular venous routes. ${ }^{14,19)}$ Transvenous embolization of the venous side of AV shunts in vein of Galen aneurysmal malformation, i.e., occlusion of the enlarged aneurysmal varix with coils is classically used as an alternative treatment. ${ }^{20,21)}$ However, we believe this approach carry a potentially high risk of hemorrhage especially in the neonatal periods, as was repeatedly pointed out by Lasjaunias. ${ }^{3)}$ In a neonatal boy with birth weight of 2,135 g (Case 3), femoral venous coil embolization to the dural ectasia was performed without control angiography because arterial access was not available. ${ }^{19)}$ Contrast injection in the dural ectasia through a microcatheter showed flow inside, from which possibility of coil occlusion of a given portion of dural ectasia had to be judged. In this neonate, temporary occlusion by the femoral venous sheath caused ipsilateral leg ischemia due to compromising venous return of the leg, and prompted quick removal of the sheath.

\section{Transcardiac approach}

Femoral and umbilical venous approaches enable not only occlusion of the venous side of AV shunts, but occlusion of the arterial side by "transcardiac approach" also as used in Cases 7 and 9. Transcardiac approach requires navigation of the balloon catheter from right atrium to left one, and then to left ventricle and ascending aorta by the experienced pediatric cardiologists. ${ }^{14,22)}$ Then, this balloon catheter is replaced with $4 \mathrm{~F}$ distal access catheter, which is further advanced into the carotid or vertebral artery. In the neonatal period, transseptal passage of the balloon catheter is through the foramen ovale, which is always widely open. Possible complications of transcardiac approach include infection, cardiac tamponade and pericardial effusion, cardiac arrest, air embolism, and esophageal varices. ${ }^{18)}$ Although we had no experience in trans-ductus arteriosus approach, it might be a theoretical option in the early neonatal period. This approach allows a catheter to enter descending aorta through ductus arteriosus. However, mechanical stimuli to the patent ductus arteriosus may close this duct rapidly, which may cause adverse effect to the neonatal cardio-pulmonary system. In addition, direction of catheter navigation is in the cranial direction, i.e., against blood flow 
in the descending aorta, which might make this approach more difficult.

\section{Conclusion}

The neonates with birth weight more than 2,700 g are commonly treated through femoral arterial routes. However, neonates with birth weight less than 2,200 g require alternative access routes other than femoral arterial routes, such as umbilical arterial/ venous, femoral venous, transcardiac, and/or direct carotid arterial access routes. Those with birth weight between 2,200 $\mathrm{g}$ and 2,700 g may also require the alternative access routes when femoral artery is too small for a $4 \mathrm{~F}$ sheath, which could be estimated by the size of the lower extremities.

\section{Acknowledgments}

The authors thank the following doctors for their referral and/or consultation of the neonates with brain arteriovenous malformations:

Dr. Hiroaki Shigeta, Department of Neurosurgery, Nagano Children's Hospital, Nagano; Dr. Yasuro Tohma, Department of Neurosurgery, Fukui Prefectural Hospital, Fukui; Dr. Shunji Matsubara, Department of Neurosurgery, Kawasaki Medical School Hospital, Okayama; Dr. Nobuyuki Hirotsune, Department of Neurosurgery, Hiroshima City Hiroshima Citizens Hospital, Hiroshima; Dr. Yasuyuki Fujita, Department of Obstetrics and Gynecology, Kyushu University Hospital, Fukuoka; Dr. Junko Yamamoto, Department of Pediatrics, Japan Community Health Care Organization Kyushu Hospital, Kitakyushu, Fukuoka; and Dr. Tatsuo Oshiro, Department of Pediatric Neonatology, Okinawa Prefectural Nanbu Medical Center and Children's Medical Center, Okinawa.

\section{Conflicts of Interest Disclosure}

The authors declare that they have no conflict of interest with regard to this manuscript, and have registered online Self-reported COI Disclosure Statement Forms through the website for the Japan Neurosurgical Society members.

\section{References}

1) Albright AL, Latchaw RE, Price RA: Posterior dural arteriovenous malformations in infancy. Neurosurgery 13: 129-135, 1983

2) Morita A, Meyer FB, Nichols DA, Patterson MC: Childhood dural arteriovenous fistulae of the posterior dural sinuses: three case reports and literature review. Neurosurgery 37: 1193-1199; discussion 1199-1200, 1995
3) Lasjaunias P: Vascular Diseases in Neonates, Infants and Children. Berlin, Springer-Verlag, 1997

4) Kincaid PK, Duckwiler GR, Gobin YP, Viñuela F: Dural arteriovenous fistula in children: endovascular treatment and outcomes in seven cases. AJNR Am J Neuroradiol 22: 1217-1225, 2001

5) Lasjaunias PL, Chng SM, Sachet M, Alvarez H, Rodesch G, Garcia-Monaco R: The management of vein of Galen aneurysmal malformations. Neurosurgery 59(5 Suppl 3): S184-S194; discussion S3-S13, 2006

6) Gold AP, Ransohoff J, Carter S: Vein of Galen malformation. Acta Neurol Scand 40 [supl 11]:5-31, 1964

7) Johnston IH, Whittle IR, Besser M, Morgan MK: Vein of Galen malformation: diagnosis and management. Neurosurgery 20: 747-758, 1987

8) Li AH, Armstrong D, terBrugge KG: Endovascular treatment of vein of Galen aneurysmal malformation: management strategy and 21-year experience in Toronto. J Neurosurg Pediatr 7: 3-10, 2011

9) Yan J, Wen J, Gopaul R, Zhang CY, Xiao SW: Outcome and complications of endovascular embolization for vein of Galen malformations: a systematic review and meta-analysis. J Neurosurg 123: 872-890, 2015

10) McSweeney N, Brew S, Bhate S, Cox T, Roebuck DJ, Ganesan V: Management and outcome of vein of Galen malformation. Arch Dis Child 95: 903-909, 2010

11) Mortensson W: Angiography of the femoral artery following percutaneous catheterization in infants and children. Acta Radiol Diagn (Stockh) 17: 581-593, 1976

12) Komiyama M, Nishikawa $M$, Yasui $T$, Kitano $S$, Sakamoto H, Inoue T: Vein of Galen aneurysmal malformation in a neonate treated by endovascular surgery-case report. Neurol Med Chir (Tokyo) 36: 893-900, 1996

13) Berenstein A, Masters LT, Nelson PK, Setton A, Verma R: Transumbilical catheterization of cerebral arteries. Neurosurgery 41: 846-850, 1997

14) Komiyama M, Nishikawa M, Kitano S, Sakamoto H, Miyagi N, Kusuda S, Sugimoto H: Transumbilical embolization of a congenital dural arteriovenous fistula at the torcular herophili in a neonate. Case report. J Neurosurg 90: 964-969, 1999

15) Komiyama M, Honnda Y, Matsusaka Y, Morikawa T, Kitano S, Sakamoto H: Cerebral diagnostic and therapeutic angiography for neonatal arteriovenous fistulas. Interv Neuroradiol 10(Suppl 1): 39-42, 2004

16) Oshiro T, Nakayama O, Ohba C, Ohashi Y, Kawakubo J, Nagamine T, Komiyama M: Transumbilical arterial embolization of a large dural arteriovenous fistula in a low-birth-weight neonate with congestive heart failure. Childs Nerv Syst 2015 [Epub ahead of print]

17) Gupta JM, Roberton NR, Wigglesworth JS: Umbilical artery catheterization in the newborn. Arch Dis Child 43: 382-387, 1968

18) Kitterman JA, Phibbs RH, Tooley WH: Catheterization of umbilical vessels in newborn infants. Pediatr Clin North Am 17: 895-912, 1970

19) Komiyama M, Matsusaka $Y$, Ishiguro $T$, Kitano $S$, Sakamoto H: Endovascular treatment of dural sinus 
malformation with arteriovenous shunt in a low birth weight neonate-case report. Neuro Med Chir (Tokyo) 44: 655-659, 2004

20) Mickle JP, Quisling RG: The transtorcular embolization of vein of Galen aneurysms. J Neurosurg 64: 731-735, 1986

21) Lylyk P, Viñuela F, Dion JE, Duckwiler G, Guglielmi G, Peacock W, Martin N: Therapeutic alternatives for vein of Galen vascular malformations. J Neurosurg 78: 438-445, 1993
22) Komiyama M, Ishiguro T, Terada A, Murakami Y: Transcardiac cerebral angiography in a child. $J$ Neurosurg Pediatr 11: 95-99, 2013

Address reprint requests to: Masaki Komiyama, MD, 2-13-22 Miyakojima-Hondori, Miyakojima, Osaka, Osaka 534-0021, Japan.

e-mail: komiyama@japan-mail.com 